\title{
ON THE ASYMPTOTIC DISTRIBUTION OF THE DISCRETE SCAN STATISTIC
}

\author{
MICHAEL V. BOUTSIKAS *** AND \\ MARKOS V. KOUTRAS, ${ }^{* * *}$ University of Piraeus
}

\begin{abstract}
The discrete scan statistic in a binary (0-1) sequence of $n$ trials is defined as the maximum number of successes within any $k$ consecutive trials ( $n$ and $k, n \geq k$, being two positive integers). It has been used in many areas of science (quality control, molecular biology, psychology, etc.) to test the null hypothesis of uniformity against a clustering alternative. In this article we provide a compound Poisson approximation and subsequently use it to establish asymptotic results for the distribution of the discrete scan statistic as $n, k \rightarrow \infty$ and the success probability of the trials is kept fixed. An extreme value theorem is also provided for the celebrated Erdôs-Rényi statistic.
\end{abstract}

Keywords: Discrete scan statistic; compound Poisson approximation; randomness test; Erdős-Rényi statistic; Kolmogorov distance; extreme value theorem

2000 Mathematics Subject Classification: Primary 62E17; 60F05

Secondary 62E20; 60F10

\section{Introduction}

Scientists dealing with experimental data modeled by independent Bernoulli trials frequently seek reasonable criteria providing clustering evidence (lack of randomness) or indicating changes in the underlying process. The length of the longest success run is definitely a very powerful statistic for studying problems of this nature, a fact that explains the continuing interest in its probabilistic characteristics since de Moivre's era (the 17th century). A natural and intuitively appealing generalization of the success run principle arises if instead of looking at pure success runs we consider the maximum number of successes within any $k$ contiguous (consecutive) trials. The resulting RV is usually referred to in the literature as the binary discrete scan statistic and has widespread applicability in a significant number of scientific areas such as quality control, molecular biology, psychology, epidemiological studies, reliability theory, etc.; see [1, pp. 377-387], [9], [12, Part I], and [8, pp. 140-151].

To fix our notation, let $X_{i}, i \in \mathbb{Z}$, be a sequence of independent, identically distributed (i.i.d.) binary random variables (RVs) with

$$
\mathrm{P}\left(X_{i}=1\right)=p, \quad \mathrm{P}\left(X_{i}=0\right)=q=1-p, \quad i=1,2, \ldots, n,
$$

and denote by

$$
S_{i}=\sum_{j=i}^{i+k-1} X_{j}, \quad i \in \mathbb{Z}
$$

Received 25 May 2005; revision received 12 July 2006.

* Postal address: Department of Statistics and Insurance Science, University of Piraeus, 80 Karaoli and Dimitriou Street, 18534 Piraeus, Greece.

** Email address: mbouts@unipi.gr

*** Email address: mkoutras@unipi.gr 
the $k$-scan process (a moving window of length $k \geq 1$ ) generated by the sequence $X_{i}, i \in \mathbb{Z}$. Then the discrete scan statistic is defined as

$$
S_{n, k}=\max _{1 \leq i \leq n-k+1} S_{i}=\max _{1 \leq i \leq n-k+1} \sum_{j=i}^{i+k-1} X_{j}
$$

where $n \geq k$ is a fixed, positive integer.

An instance where $S_{n, k}$ arises in quite a natural way is in randomness tests when the null hypothesis of uniformity and independence of $X_{i}, i=1,2, \ldots, n$, is to be tested against the alternative hypothesis of clustering of $1 \mathrm{~s}$ due to local positive dependence between $X_{i}, i=$ $1,2, \ldots, n$, or due to the existence of subsequences of consecutive $X_{i}$ with $\mathrm{P}\left(X_{i}=1\right)>p$. As Glaz and Naus [10] indicated, the generalized likelihood ratio test for checking the hypothesis of uniformity rejects the null hypothesis of uniformity whenever $S_{n, k} \geq c$, with the value of $c$ being determined by the significance level of the test. Recently, Glaz and Zhang [11] introduced an alternative, more sensitive, procedure exploiting a multiple scan statistic of variable size instead of the single (fixed window length) scan statistic $S_{n, k}$.

Apparently, the evaluation of $c$ such that a prespecified significance level is achieved calls for the distribution of the test statistic $S_{n, k}$. Since randomness tests are frequently applied to large data sets, theoretical developments related to the asymptotic distribution of $S_{n, k}$ (as $\left.n, k \rightarrow \infty\right)$ will play a primary role in the analysis of the test.

Another instance where $S_{n, k}$ could be used is offered by the following model, which originates in molecular biology. In the study of amino acid sequences, various classification schemes are in common use, including a chemical alphabet of eight letters, a functional alphabet of four letters, a charge alphabet of three letters, etc. In order to introduce quantitative means for assessing and interpreting genomic inhomogeneities between sequences of different species or sequences subject to different chemical infections and/or several levels of corruption, molecular biologists look for long aligned subsequences that match in most of their positions, and try to specify what is an unusually long match. In order to construct an appropriate mathematical model, let $Z_{i 1}$ and $Z_{i 2}, i=1,2, \ldots, n$, be two amino acid sequences from a finite alphabet $A=\left\{a_{1}, a_{2}, \ldots, a_{l}\right\}$, with $\mu_{j}=\mathrm{P}\left(Z_{i 1}=a_{j}\right)=\mathrm{P}\left(Z_{i 2}=a_{j}\right), j=1,2, \ldots, l$. The two sequences will be said to match in position $i \in\{1,2, \ldots, n\}$ if $Z_{i 1}=Z_{i 2}$, in which case we let $X_{i}$ be 1 (we let $X_{i}$ be 0 otherwise). Then $X_{i}, i=1,2, \ldots, n$, form a sequence of binary i.i.d. RVs with success probabilities

$$
p=\mathrm{P}\left(X_{i}=1\right)=\mathrm{P}\left(Z_{i 1}=Z_{i 2}\right)=\sum_{j=1}^{l} \mu_{j}^{2},
$$

and the number of matches over a window of length $k$ will be described by the corresponding $k$-scan process $S_{i}, i=1,2, \ldots, n$. Moreover, a 'near perfect' match at position $i$ can be described by the event $S_{i} \geq c$, with $c$ being an integer sufficiently close to $k$. It is clear that the condition

$$
S_{n, k}=\max _{1 \leq i \leq n-k+1} S_{i}<c
$$

can then be used as evidence of the lack of a local match between the two sequences under inspection. It should be stressed that, in this application, we are also interested in large values of both $n$ (long amino acid sequences) and $k$ (long matching regions). 
As a final example we provide the following actuarial model. Let $Z_{i}, i=1,2, \ldots, n$, be the daily claim sizes over an $n$-day period and $u \geq 0$ a given threshold. Assume that the $Z_{i}$ are i.i.d. RVs with cumulative distribution function $F$, and denote by

$$
X_{i}=\mathbf{1}_{(u, \infty)}\left(Z_{i}\right)=\left\{\begin{array}{ll}
1 & \text { if } Z_{i}>u, \\
0 & \text { if } Z_{i} \leq u,
\end{array} \quad i=1,2, \ldots, n,\right.
$$

the corresponding RVs, which indicate whether or not the $i$ th claim exceeds the threshold $u$. (Here $\mathbf{1}_{A}(\cdot)$ denotes the indicator function of the (generic) set $A$.) Then

$$
\mathrm{P}\left(X_{i}=1\right)=\mathrm{E}\left(X_{i}\right)=\mathrm{P}\left(Z_{i}>u\right)=1-F(u)=p, \quad i=1,2, \ldots, n,
$$

and $S_{n, k}$ will describe the maximum number of 'large claims' (i.e. claims exceeding the threshold $u$ ) in a period of $k$ consecutive days. Since the primary interest in this situation is also focused on extremely long periods $(n \rightarrow \infty, k \rightarrow \infty)$, one should look at the asymptotic distribution of $S_{n, k}$.

In all the aforementioned examples, it is clear that the study of the underlying model calls for the investigation of the distribution of the RV $S_{n, k}$. Exact results for the distribution of the scan statistic were discussed in [7], [1, pp. 291-301], and [8, pp. 88-96]. Since the evaluation of the exact distribution is computationally intractable, especially for large values of the parameters, several approximations and bounds have been developed during the last decade. The interested reader may refer to the recent monographs by Glaz et al. [12] and Balakrishnan and Koutras [1] for up-to-date reviews of this topic.

In a recent article by Boutsikas and Koutras [4], a compound Poisson approximation was established for the distribution of the enumerating RV

$$
W_{n}=\sum_{i=1}^{n-k+1} \mathbf{1}_{[r, \infty)}\left(S_{i}\right)
$$

As a by-product, an approximation for

$$
\mathrm{P}\left(S_{n, k}<r\right)=\mathrm{P}\left(W_{n}=0\right)
$$

was established, along with an upper bound for the error incurred in its use. However, the asymptotic result given there holds under the conditions $n \rightarrow \infty, p \rightarrow 0$ with $k$ and $r$ fixed, which are of no interest in the examples mentioned above. One might suspect that, even in the case of interest ( $p$ fixed and $n, k \rightarrow \infty$ ) a compound Poisson law underlies the behavior, yet the tests for this provided by the results of [4] are inconclusive. This is due to the fact that, for $r<k$, the upper bound appearing there is of order $O(p)$ and, therefore, does not converge to 0 as $n, k \rightarrow \infty$ while $p$ is fixed.

In the present article, motivated by the abovementioned remarks, we establish a new compound Poisson approximation for $W_{n}$ that offers an upper bound manageable under the conditions of interest.

In Section 2 we introduce all necessary notation and preliminary material. In Section 3, exploiting an appropriate declumping technique, we develop a compound Poisson approximation for the distribution of $W_{n}$, along with tight upper bounds for the Kolmogorov distance between the distribution of $W_{n}$ and the approximating distribution. In Section 4 an asymptotic result for the distribution of the scan statistic $S_{n, k}$ is established, while in Section 5 we present 
an extreme value theorem for the same statistic that is comparable to the well-known ErdôsRényi results (when applied to binary sequences). Finally, in Section 6 an extensive numerical experimentation is carried out in order to investigate the quality of the approximations and bounds.

\section{Preliminaries}

The Kolmogorov distance between the distributions of two RVs $X$ and $Y$ is defined as

$$
d(X, Y)=\sup _{w}|\mathrm{P}(X \leq w)-\mathrm{P}(Y \leq w)|
$$

and offers a very efficient tool for establishing convergence in distribution; a sequence of RVs converges weakly to $Y$ if the corresponding sequence of distances converges to 0 . By the term 'compound Poisson distribution $\mathrm{CP}(\lambda, H)$ with parameter $\lambda$ and compounding distribution $H$ ', we shall refer to the distribution of a random sum of the form $\sum_{i=1}^{N} Z_{i}$ where $N$ is a Poisson RV with $\lambda=\mathrm{E}(N)$ and the $Z_{i}$ are i.i.d. RVs (also independent of $N$ ) whose distribution function is $H$.

The main result of the next section is an application of a general theorem on compound Poisson approximation published by Boutsikas and Koutras [3]. For the purposes of the present exposition, we shall retain a simplified version of their result, which is more than adequate to meet our needs.

Consider first a sequence of nonnegative RVs $Z_{a}, a=1,2, \ldots$ For each $a=2,3, \ldots$, introduce a subset, $B_{a}$, of $\{1,2, \ldots, a-1\}$ (the left neighborhood of dependence of $Z_{a}$ ) such that $Z_{a}$ is independent of all $Z_{b}, b \in\{1,2, \ldots, a-1\} \backslash B_{a}$. The next theorem provides an upper bound for the Kolmogorov distance between the distribution of the sum $\sum_{a=1}^{v} Z_{a}$ (with $v$ a fixed, positive integer) and a compound Poisson distribution $\mathrm{CP}(\lambda, H)$ with suitably chosen $\lambda$ and $H$.

Theorem 1. (Boutsikas and Koutras [3].) If $Z_{a}, a=1,2, \ldots, v$, is a sequence of nonnegative $R V s$, then

$$
\begin{aligned}
d\left(\sum_{a=1}^{v} Z_{a}, \mathrm{CP}(\lambda, H)\right) \leq & \sum_{a=2}^{v}\left(\mathrm{P}\left(Z_{a}>0, \sum_{b \in B_{a}} Z_{b}>0\right)+\mathrm{P}\left(Z_{a}>0\right) \mathrm{P}\left(\sum_{b \in B_{a}} Z_{b}>0\right)\right) \\
& +\frac{1}{2} \sum_{i=1}^{v} \mathrm{P}\left(Z_{i}>0\right)^{2}
\end{aligned}
$$

where $\lambda=\sum_{a=1}^{v} \lambda_{a}$ and

$$
H(x)=\frac{1}{\lambda} \sum_{a=1}^{v} \lambda_{a} \mathrm{P}\left(Z_{a} \leq x \mid Z_{a}>0\right), \quad x \in \mathbb{R},
$$

with $\lambda_{a}=\mathrm{P}\left(Z_{a}>0\right), a=1,2, \ldots, v$.

Theorem 1 states that if the RVs $Z_{a}, a=1,2, \ldots$, are 'locally' dependent and the masses of their distributions are concentrated on 0 , then $\sum_{a=1}^{v} Z_{a}$ can be satisfactorily approximated by an appropriate compound Poisson distribution. 
If $X$ and $Y$ are nonnegative RVs, it is evident that $|\mathrm{P}(X=0)-\mathrm{P}(Y=0)| \leq d(X, Y)$ and, therefore, that

$$
\left|\mathrm{P}\left(\sum_{a=1}^{v} Z_{a}=0\right)-\mathrm{e}^{-\lambda}\right|
$$

is also bounded from above by the right-hand side of (2). It is worth stressing that, should one wish to establish bounds for $\mathrm{P}\left(\sum_{a=1}^{v} Z_{a}=0\right)$ only (and not for the whole distribution of $\sum_{a=1}^{v} Z_{a}$ ), there is no need to proceed to the calculation of the compounding distribution $H$.

Now let $b(x ; n, p)$ and $B(x ; n, p)$ respectively denote the probability mass function and cumulative distribution function of a binomial RV $X$, i.e.

$$
\begin{array}{ll}
b(x ; n, p)=\mathrm{P}(X=x)=\left(\begin{array}{l}
n \\
x
\end{array}\right) p^{x} q^{n-x}, & x=0,1, \ldots, n, \\
B(x ; n, p)=\mathrm{P}(X \leq x)=\sum_{r=0}^{x} b(r ; n, p), & x \in \mathbb{R} .
\end{array}
$$

In the following sections we shall make frequent use of the quantities

$$
\begin{aligned}
& f(s ; k, p)=\mathrm{P}\left(S_{1}<s, S_{2}<s, \ldots, S_{k}<s, S_{k+1} \geq s\right), \\
& G(s ; k, p)=\mathrm{P}\left(S_{1}<s, S_{2}<s, \ldots, S_{k+1}<s\right),
\end{aligned}
$$

which can be expressed using $b(x ; n, p)$ and $B(x ; n, p)$ as follows (cf. [10]), for $1 \leq s \leq k$ (if $s>k$ or $s<0$ then we set $f(s, k ; p)=0)$ :

$$
\begin{aligned}
f(s ; k, p) & =\frac{p}{s} b(s-1 ; k-1, p)[s q b(s-1 ; k-1, p)+(s-k p) B(s-2 ; k-1, p)], \\
G(s ; k, p) & =B(s-1 ; k, p)^{2}-b(s ; k, p)[(s-1) B(s-2 ; k, p)-k p B(s-3 ; k-1, p)] .
\end{aligned}
$$

The standard symbols ' $\sim$ ', $O(\cdot)$, and $O(\cdot)$ will assume their usual meanings, i.e.

$$
\begin{aligned}
& f(t) \sim g(t) \quad \text { as } t \rightarrow t_{0} \quad \text { if } \lim _{t \rightarrow t_{0}} \frac{f(t)}{g(t)}=1, \\
& f(t)=o(g(t)) \quad \text { as } t \rightarrow t_{0} \quad \text { if } \lim _{t \rightarrow t_{0}} \frac{f(t)}{g(t)}=0, \\
& f(t)=O(g(t)) \quad \text { if } \frac{f(t)}{g(t)} \text { is bounded. }
\end{aligned}
$$

In addition, summations of the form $\sum_{i=a}^{b} x_{i}$ with $a>b$ will be assumed to vanish. Finally, we shall write $\lfloor x\rfloor$ for the integer part of $x$.

\section{An approximation for the cumulative distribution function of $S_{n, k}$}

As stated after Theorem 1, should we wish to exploit (2) to establish fine upper bounds (i.e. bounds converging to 0$)$ for $d\left(W_{n}, \mathrm{CP}(\lambda, H)\right.$ ) or simply for

$$
\begin{aligned}
\left|\mathrm{P}\left(S_{n, k}<r\right)-\mathrm{e}^{-\lambda}\right| & =\left|\mathrm{P}\left(W_{n}=0\right)-\mathrm{e}^{-\lambda}\right| \\
& =\mid \mathrm{P}\left(\mathbf{1}_{[r, \infty)}\left(S_{a}\right)=0 \text { for all } a=1, \ldots, n-k+1\right)-\mathrm{e}^{-\lambda} \mid,
\end{aligned}
$$


care should be taken that $Z_{a}, a=1,2, \ldots$, are locally dependent and have probability mass functions concentrated on 0 . Since scans exhibit a strong tendency to cluster (especially if $p$ does not converge to 0$)$, a direct application of Theorem 1 with $Z_{a}=\mathbf{1}_{[r, \infty)}\left(S_{a}\right), a=$ $1,2, \ldots$, does not yield powerful estimates for the approximation error. One convenient way to improve the performance of the upper bound is to first introduce a set of weakly dependent RVs $Z_{a}=C_{a}, a=1,2, \ldots$, so a small upper bound is gained for $d\left(\sum Z_{a}, \mathrm{CP}(\lambda, H)\right)$ through Theorem 1, and then make use of the triangle inequality

$$
d\left(W_{n}, \mathrm{CP}(\lambda, H)\right) \leq d\left(W_{n}, \sum Z_{a}\right)+d\left(\sum Z_{a}, \mathrm{CP}(\lambda, H)\right) .
$$

It goes without saying that an efficient upper bound for the quantity $d\left(W_{n}, \sum Z_{a}\right)$ will also be needed.

A set of RVs possessing the aforementioned properties is provided by

$$
C_{a}=\left[\prod_{j=a-k}^{a-1}\left(1-\mathbf{1}_{[r, \infty)}\left(S_{j}\right)\right)\right]\left[\mathbf{1}_{[r, \infty)}\left(S_{a}\right) \sum_{m=a}^{a+k} \mathbf{1}_{[r, \infty)}\left(S_{m}\right)\right], \quad a=1,2, \ldots
$$

The second bracket enumerates the number of scanning windows of length $k$ that begin at positions $a, a+1, \ldots, a+k$ and contain at least $r$ successes each (such a RV, which counts the total number of clumps located in a specific area, is usually called a declumping variable). On the other hand, the first bracket guarantees that at the previous $k$ positions, $a-k, a-k+1, \ldots, a-1$, all scanning windows of length $k$ contain fewer than $r$ successes. As a matter of fact, it is the inclusion of this extra term that makes the construction of sharp bounds feasible; were we to represent the declumping procedure exclusively by the second bracket and the last term of the first bracket, then the resulting bounds would exhibit a slow convergence rate (of order $O(p)$ ) for $r<k$, and only the case $r=k$ could exhibit a better rate of order $O\left(p^{k}\right)$. For more details on this approach we refer the reader to [4]. We are now ready to prove the next theorem.

Theorem 2. Let $W_{n}=\sum_{i=1}^{n-k+1} \mathbf{1}_{[r, \infty)}\left(S_{i}\right)$ be the number of moving sums that contain at least $r$ 1s. Then

$$
\begin{aligned}
& d\left(W_{n}, \mathrm{CP}(\lambda, H)\right) \\
& \quad \leq(2 k-1) \lambda p q b(r-1 ; k-1, p)+3 \lambda k f(r ; k, p)+(\lambda+2)(1-G(r ; k, p)),
\end{aligned}
$$

where $\lambda \equiv \lambda_{r, k, n}=(n-k+1) f(r ; k, p)$ and

$$
\begin{aligned}
H(x) & =\mathrm{P}\left(C_{1} \leq x \mid C_{1}>0\right) \\
& =\mathrm{P}\left(\sum_{m=k+1}^{2 k+1} \mathbf{1}_{[r, \infty)}\left(S_{m}\right) \leq x \mid \mathbf{1}_{[r, \infty)}\left(S_{j}\right)=0, j=1,2, \ldots, k, \mathbf{1}_{[r, \infty)}\left(S_{k+1}\right)=1\right) .
\end{aligned}
$$

Proof. By applying inequality (5) with $Z_{a}=C_{a}, a=1,2, \ldots$, we may write

$$
d\left(W_{n}, \mathrm{CP}(\lambda, H)\right) \leq d\left(W_{n}, \sum_{a=1}^{n-k+1} C_{a}\right)+d\left(\sum_{a=1}^{n-k+1} C_{a}, \mathrm{CP}(\lambda, H)\right)
$$


where (see also (3) and (4))

$$
\lambda=\sum_{a=1}^{n-k+1} \mathrm{P}\left(C_{a}>0\right)=(n-k+1) f(r ; k, p) .
$$

The second term on the right-hand side of (6) can be bounded from above with the aid of Theorem 1. More specifically, if we introduce

$$
B_{a}=\{\max \{1, a-3 k+1\}, \ldots, a-1\}, \quad a=2,3, \ldots,
$$

the left neighborhoods of dependence, we deduce that

$$
\begin{aligned}
d\left(\sum_{a=1}^{n-k+1} C_{a}, \mathrm{CP}(\lambda, H)\right) & \\
\leq & \sum_{i=2}^{n-k+1} \sum_{b=\max \{1, i-3 k+1\}}^{i-1}\left(\mathrm{P}\left(C_{b}>0, C_{i}>0\right)+\mathrm{P}\left(C_{b}>0\right) \mathrm{P}\left(C_{i}>0\right)\right) \\
& +(n-k+1) \mathrm{P}\left(C_{1}>0\right)^{2} \\
& \leq \sum_{i=2}^{n-k+1} \sum_{b=\max \{1, i-3 k+1\}}^{i-k-1} \mathrm{P}\left(C_{b}>0, C_{i}>0\right)+3 k(n-k+1) \mathrm{P}\left(C_{1}>0\right)^{2} \\
\leq & (n-k) \sum_{b=1}^{2 k-1} \mathrm{P}\left(C_{b}>0, C_{3 k}>0\right)+3(n-k+1) k f^{2}(r ; k, p) \\
\leq & (n-k) \sum_{b=1}^{2 k-1} \mathrm{P}\left(S_{b-k}<r, \ldots, S_{b-1}<r, S_{b} \geq r\right) \mathrm{P}\left(S_{3 k-1}<r, S_{3 k} \geq r\right) \\
& +3(n-k+1) k f^{2}(r ; k, p) \\
\leq & \lambda(2 k-1)\left(\begin{array}{l}
k-1 \\
r-1
\end{array}\right) p^{r} q^{k-r+1}+3 \lambda k f(r ; k, p) .
\end{aligned}
$$

On the other hand, for the first term of (6) we have (using the well-known coupling inequality for the total variation distance, $d_{\mathrm{TV}}$ )

$$
d\left(W_{n}, \sum_{a=1}^{n-k+1} C_{a}\right) \leq d_{\mathrm{TV}}\left(W_{n}, \sum_{a=1}^{n-k+1} C_{a}\right) \leq \mathrm{P}\left(W_{n} \neq \sum_{a=1}^{n-k+1} C_{a}\right) .
$$

The RVs $W_{n}$ and $\sum_{i=1}^{n-k+1} C_{i}$ are unequal only in the following three cases.

(i) A loose clump that starts at trial $i$ does not end until trial $i+2 k-1, i=1,2, \ldots, n-2 k$.

(ii) One of the scanning windows starting at $n-k+2, \ldots, n+1$ contains at least $r 1 \mathrm{~s}$.

(iii) One of the scanning windows starting at $-k+1, \ldots, 0$ contains at least $r 1 \mathrm{~s}$. 
Cases (ii) and (iii) occur because of so-called 'edge effects', while case (i) occurs because, for computational convenience, we used truncated clumps. Hence, the following inequality will hold:

$$
\begin{aligned}
d\left(W_{n}, \sum_{i=1}^{n-k+1} C_{i}\right) \leq & \sum_{i=1}^{n-2 k} \mathrm{P}\left(C_{i}>0, S_{i+k+1} \geq r \text { or } S_{i+k+2} \geq r \text { or } \cdots \text { or } S_{i+2 k} \geq r\right) \\
& +2\left(1-\mathrm{P}\left(S_{1}<r, \ldots, S_{k}<r\right)\right) \\
\leq & \sum_{i=1}^{n-2 k} \mathrm{P}\left(S_{i-k}<r, \ldots, S_{i-1}<r, S_{i} \geq r\right) \\
& \times\left(1-\mathrm{P}\left(S_{i+k+1}<r, \ldots, S_{i+2 k+1}<r\right)\right) \\
& +2\left(1-\mathrm{P}\left(S_{1}<r, \ldots, S_{k}<r, S_{k+1}<r\right)\right) \\
= & (n-2 k) f(r ; k, p)(1-G(r ; k, p))+2(1-G(r ; k, p)) \\
\leq & (\lambda+2)(1-G(r ; k, p)) .
\end{aligned}
$$

This concludes the proof of the theorem.

The following corollary is an immediate consequence of the above theorem and (1).

Corollary 1. Let $F_{n, k}(r)=\mathrm{P}\left(S_{n, k}<r\right), r=1,2, \ldots, k$, denote the cumulative distribution function of the discrete scan statistic $S_{n, k}$. Then

$\left|F_{n, k}(r)-\mathrm{e}^{-\lambda}\right| \leq(2 k-1) \lambda p q b(r-1 ; k-1, p)+3 \lambda k f(r ; k, p)+(\lambda+2)(1-G(r ; k, p))$,

where $\lambda \equiv \lambda_{r, k, n}=(n-k+1) f(r ; k, p)$.

Roos [15] has developed several results that can be used to establish compound Poisson approximations for sums of dependent RVs (see also [2] for additional references on this topic). For the problem at hand, it is unclear whether these results can be profitably exploited to produce as manageable an upper bound as the one given in Theorem 2. Moreover, even if such a bound was established, it is not expected to improve on the order of convergence offered by our result.

\section{The asymptotic distribution of $S_{n, k}$}

In the present section we are going to present a large deviation result for $S_{n, k}$. Let us first introduce some additional notation that will be used in the sequel.

For $0<p<\theta<1$ we shall denote by $H(\theta, p)$ the relative entropy (of the Bernoulli distribution with parameter $\theta$ with respect to the Bernoulli distribution with parameter $p$ ) or Kullback-Leibler distance, which is given by

$$
H(\theta, p)=\theta \ln \frac{\theta}{p}+(1-\theta) \ln \frac{1-\theta}{1-p}=\ln \frac{\theta^{\theta}(1-\theta)^{1-\theta}}{p^{\theta}(1-p)^{1-\theta}} .
$$

The derivative of $H(\theta, p)$ with respect to $\theta$,

$$
h(\theta, p)=\frac{\mathrm{d}}{\mathrm{d} \theta} H(\theta, p)=\ln \left(\frac{\theta /(1-\theta)}{p /(1-p)}\right)>0,
$$


measures the log-odds ratio between two biased coins. It is clear that $H(\theta, p)$ increases from 0 to $\ln (1 / p)$ as $\theta$ increases from $p$ to 1 .

We shall now present a simple auxiliary lemma that will prove useful in the investigation of the asymptotic distribution of $S_{n, k}$. Henceforth, we shall assume that $r \equiv r_{n}$ and $k \equiv k_{n}$, with both sequences, $\left\{r_{n}\right\}$ and $\left\{k_{n}\right\}$, tending to $\infty$ as $n \rightarrow \infty$. Where not stated explicitly, all convergences and limits apply as $n \rightarrow \infty$.

Lemma 1. If $p$ is fixed, $\theta \in(p, 1)$, and $r_{n}$ and $k_{n}$ satisfy the condition

$$
\lim \frac{r_{n}-\theta k_{n}}{\sqrt{k_{n}}}=0
$$

then

$$
\begin{aligned}
\left(\begin{array}{l}
k \\
r
\end{array}\right) \theta^{r}(1-\theta)^{k-r} & =\frac{1+O\left(\left(\rho^{2}+1\right) / k\right)}{\sqrt{2 \pi \theta(1-\theta) k}} \\
\sum_{i=r}^{k}\left(\begin{array}{l}
k \\
i
\end{array}\right) p^{i}(1-p)^{k-i} & \sim \frac{\theta(1-p)}{\theta-p} \frac{\mathrm{e}^{-k H(\theta, p)-\rho h(\theta, p)}}{\sqrt{2 \pi \theta(1-\theta) k}},
\end{aligned}
$$

where $\rho \equiv \rho_{n}=r_{n}-\theta k_{n}=o\left(\sqrt{k_{n}}\right)$.

Proof. Note first that, for any sequence $\left\{a_{n}\right\}$ of real numbers with $a_{n}=o\left(\sqrt{k_{n}}\right)$, we have

$$
\frac{\left(1-a_{n} / k_{n}\right)^{k_{n}}}{\mathrm{e}^{-a_{n}}}=1+O\left(\frac{a_{n}^{2}}{k_{n}}\right) \rightarrow 1 .
$$

This is readily ascertainable if we apply the elementary inequality $x \leq-\ln (1-x) \leq x /(1-x)$ with $x=a_{n} / k_{n}<1$ (note that $\lim _{n \rightarrow \infty}\left(a_{n} / k_{n}\right)=0$ and assume that $k_{n}$ is large enough that $\left.a_{n} / k_{n}<1\right)$, to obtain

$$
\exp \left(-\frac{1}{1-a_{n} / k_{n}} \frac{a_{n}^{2}}{k_{n}}\right) \leq \frac{\left(1-a_{n} / k_{n}\right)^{k_{n}}}{\mathrm{e}^{-a_{n}}} \leq 1 .
$$

In view of the last inequality we may write

$$
\left|1-\frac{\left(1-a_{n} / k_{n}\right)^{k_{n}}}{\mathrm{e}^{-a_{n}}}\right| \leq 1-\exp \left(-\frac{1}{1-a_{n} / k_{n}} \frac{a_{n}^{2}}{k_{n}}\right)=O\left(\frac{a_{n}^{2}}{k_{n}}\right),
$$

which proves the asymptotic expression (10).

Next, a straightforward application of Stirling's formula yields

$$
\begin{aligned}
\left(\begin{array}{l}
k \\
r
\end{array}\right) & =\frac{k^{k} \sqrt{2 \pi k} \mathrm{e}^{c_{k} / 12 k}}{\mathrm{e}^{k}} \frac{\mathrm{e}^{r}}{r^{r} \sqrt{2 \pi r} \mathrm{e}^{c_{r} / 12 r}} \frac{\mathrm{e}^{k-r}}{(k-r)^{k-r} \sqrt{2 \pi(k-r)} \exp \left(c_{k-r} /[12(k-r)]\right)} \\
& =\frac{1}{\sqrt{2 \pi r(1-r / k)}} \frac{k^{k}}{r^{r}(k-r)^{k-r}} \exp \left(\frac{1}{12 k}\left(c_{k}-\frac{c_{r}}{r / k}-\frac{c_{k-r}}{1-r / k}\right)\right),
\end{aligned}
$$

where $c_{i} \in(0,1), i=1,2, \ldots$ By making use of the obvious equality

$$
\frac{k^{k}}{r^{r}(k-r)^{k-r}} \theta^{r}(1-\theta)^{k-r}=\left(\frac{\theta k}{r}\right)^{r}\left(\frac{(1-\theta) k}{k-r}\right)^{k-r}
$$


and taking into account the asymptotic expansions

$$
\begin{gathered}
\left(\frac{\theta k}{r}\right)^{r}=\left(1+\frac{\theta k-r}{r}\right)^{r}=\left(1-\frac{\rho}{r}\right)^{r}=\mathrm{e}^{-\rho}\left(1+O\left(\frac{\rho^{2}}{k}\right)\right) \\
\left(\frac{(1-\theta) k}{k-r}\right)^{k-r}=\left(1+\frac{r-k \theta}{k-r}\right)^{k-r}=\left(1+\frac{\rho}{k-r}\right)^{k-r}=\mathrm{e}^{\rho}\left(1+O\left(\frac{\rho^{2}}{k}\right)\right)
\end{gathered}
$$

(resulting from (10) with $a_{n}=\rho_{n} k_{n} / r_{n}$ and $a_{n}=\rho_{n} k_{n} /\left(k_{n}-r_{n}\right)$, respectively), we conclude that

$$
\begin{aligned}
\left(\begin{array}{l}
k \\
r
\end{array}\right) \theta^{r} & (1-\theta)^{k-r} \\
& =\frac{1}{\sqrt{2 \pi r(1-r / k)}}\left(\frac{\theta k}{r}\right)^{r}\left(\frac{(1-\theta) k}{k-r}\right)^{k-r} \exp \left(\frac{1}{12 k}\left(c_{k}-\frac{c_{r}}{r / k}-\frac{c_{k-r}}{1-r / k}\right)\right) \\
& =\frac{\left(1+O\left(\rho^{2} / k\right)\right)}{\sqrt{2 \pi r(1-r / k)}} \exp \left(\frac{1}{12 k}\left(c_{k}-\frac{c_{r}}{r / k}-\frac{c_{k-r}}{1-r / k}\right)\right) .
\end{aligned}
$$

The proof of (8) is now easily completed by observing that

$$
\begin{aligned}
\sqrt{\frac{\theta(1-\theta)}{(r / k)(1-r / k)}}-1 & =O\left(\frac{\rho}{k}\right), \\
\exp \left(\frac{1}{12 k}\left(c_{k}-\frac{c_{r}}{r / k}-\frac{c_{k-r}}{1-r / k}\right)\right)-1 & =O\left(\frac{1}{k}\right) .
\end{aligned}
$$

To prove (9), first note that

$$
\frac{\sum_{i=r}^{k}\left(\begin{array}{l}
k \\
i
\end{array}\right) p^{i} q^{k-i}}{\left(\begin{array}{l}
k \\
r
\end{array}\right) p^{r} q^{k-r}}=1+\sum_{i=1}^{k-r} \frac{(k-r)(k-r-1) \cdots(k-r-i+1)}{(r+1)(r+2) \cdots(r+i)}\left(\frac{p}{q}\right)^{i} \leq \sum_{i=0}^{k-r}\left(\frac{k-r}{r} \frac{p}{q}\right)^{i}
$$

$(q=1-p)$. Since $r / k \rightarrow \theta>p$, we may choose $r$ and $k$ to be large enough that

$$
\frac{k-r}{r} \frac{p}{q}=\frac{1-r / k}{r / k} \frac{p}{1-p}<1
$$

whence

$$
\frac{\sum_{i=r}^{k}\left(\begin{array}{l}
k \\
i
\end{array}\right) p^{i} q^{k-i}}{\left(\begin{array}{l}
k \\
r
\end{array}\right) p^{r} q^{k-r}} \leq \frac{1-([(k-r) / r] p / q)^{k-r+1}}{1-[(k-r) / r] p / q} \rightarrow \frac{1}{1-[(1-\theta) / \theta] p / q}=\frac{\theta-\theta p}{\theta-p} .
$$

Observe next that, for $k$ and $r$ large enough that

$$
\frac{k-r-\lfloor\sqrt{k-r}\rfloor}{r+\lfloor\sqrt{k-r}\rfloor} \frac{p}{1-p}<1
$$


we may write

$$
\begin{aligned}
\frac{\sum_{i=r}^{k}\left(\begin{array}{c}
k \\
i
\end{array}\right) p^{i} q^{k-i}}{\left(\begin{array}{l}
k \\
r
\end{array}\right) p^{r} q^{k-r}} & \geq 1+\sum_{i=1}^{\lfloor\sqrt{k-r}\rfloor} \frac{(k-r)(k-r-1) \cdots(k-r-i+1)}{(r+1)(r+2) \cdots(r+i)}\left(\frac{p}{q}\right)^{i} \\
& \geq \sum_{i=0}^{\lfloor\sqrt{k-r}\rfloor}\left(\frac{k-r-\lfloor\sqrt{k-r}\rfloor}{r+\lfloor\sqrt{k-r}\rfloor}\right)^{i}\left(\frac{p}{q}\right)^{i} \\
& =\frac{1-((k-r-\lfloor\sqrt{k-r}\rfloor) /(r+\lfloor\sqrt{k-r}\rfloor)(p / q))^{\lfloor\sqrt{k-r}\rfloor+1}}{1-(k-r-\lfloor\sqrt{k-r}\rfloor) /(r+\lfloor\sqrt{k-r}\rfloor)(p / q)} \\
& \rightarrow \frac{1}{1-[(1-\theta) / \theta] p / q}=\frac{\theta-\theta p}{\theta-p} .
\end{aligned}
$$

Hence,

$$
\frac{\sum_{i=r}^{k}\left(\begin{array}{c}
k \\
i
\end{array}\right) p^{i} q^{k-i}}{\left(\begin{array}{l}
k \\
r
\end{array}\right) p^{r} q^{k-r}} \rightarrow \frac{\theta-\theta p}{\theta-p},
$$

and the proof is easily completed if we use (8) and take into account the fact that

$$
\mathrm{e}^{-k H(\theta, p)-\rho h(\theta, p)}=\frac{p^{r}(1-p)^{k-r}}{\theta^{r}(1-\theta)^{k-r}} .
$$

It is worth mentioning that (9) can be viewed as a special case of Petrov's [14] well-known large deviation theorem (see also [13] for an extension of Petrov's result).

We are now ready to elucidate the asymptotic behavior of $S_{n, k}$.

Theorem 3. Let $p$ be fixed, let $\theta \in(p, 1)$, and let $\left\{r_{n}\right\}$ and $\left\{k_{n}\right\}$ be two sequences satisfying the condition

$$
\lim _{n \rightarrow \infty} \frac{r_{n}-\theta k_{n}}{\sqrt{k_{n}}}=0
$$

If the sequence

$$
l_{n}=n \frac{(\theta-p) \mathrm{e}^{-k H(\theta, p)-\rho h(\theta, p)}}{\sqrt{2 \pi \theta(1-\theta) k}}, \quad n=1,2, \ldots
$$

$\left(\rho \equiv \rho_{n}=r_{n}-\theta k_{n}\right)$, is bounded from above, then

$$
\mathrm{P}\left(S_{n, k}<r\right) \sim \mathrm{e}^{-l_{n}} .
$$

Moreover, the rate of convergence in the above approximation is of order $O\left(\left(\rho^{2}+1\right) / k\right)$.

Proof. Recalling the notation used in Corollary 1, we may write

$$
\left|\mathrm{P}\left(S_{n, k}<r\right)-\mathrm{e}^{-l_{n}}\right| \leq\left|F_{n, k}(r)-\mathrm{e}^{-\lambda_{r, k, n}}\right|+\left|\mathrm{e}^{-\lambda_{r, k, n}}-\mathrm{e}^{-l_{n}}\right| .
$$

By (8), we deduce that

$$
\begin{aligned}
f(r ; k, p) & =\frac{r}{k}\left(\begin{array}{l}
k \\
r
\end{array}\right) p^{r} q^{k-r}\left[\frac{q r}{p k}\left(\begin{array}{l}
k \\
r
\end{array}\right) p^{r} q^{k-r}+\left(1-\frac{k p}{r}\right)\left(1-\sum_{i=r}^{k} \frac{i}{k p}\left(\begin{array}{l}
k \\
i
\end{array}\right) p^{i} q^{k-i}\right)\right] \\
& =\frac{(\theta-p) \mathrm{e}^{-k H(\theta, p)-\rho h(\theta, p)}}{\sqrt{2 \pi \theta(1-\theta) k}}\left(1+O\left(\frac{\rho^{2}+1}{k}\right)\right),
\end{aligned}
$$


while (9) yields

$$
\begin{aligned}
1-G(r ; k, p)= & 1-\left(1-\sum_{i=r}^{k}\left(\begin{array}{l}
k \\
i
\end{array}\right) p^{i} q^{k-i}\right)^{2}+k p\left(\begin{array}{l}
k \\
r
\end{array}\right) p^{r} q^{k-r} \\
& \times\left(\frac{r-1-p k}{p k}-\frac{r-1}{p k} \sum_{i=r-1}^{k}\left(\begin{array}{c}
k \\
i
\end{array}\right) p^{i} q^{k-i}+\sum_{i=r-2}^{k-1}\left(\begin{array}{c}
k-1 \\
i
\end{array}\right) p^{i} q^{k-1-i}\right) \\
& \sim 2 \frac{\theta(1-p)}{\theta-p} \frac{\mathrm{e}^{-k H(\theta, p)-\rho h(\theta, p)}}{\sqrt{2 \pi \theta(1-\theta) k}}+k p \frac{\mathrm{e}^{-k H(\theta, p)-\rho h(\theta, p)}}{\sqrt{2 \pi \theta(1-\theta) k}} \frac{\theta-p}{p} \\
& \sim \frac{(\theta-p) k \mathrm{e}^{-k H(\theta, p)-\rho h(\theta, p)}}{\sqrt{2 \pi \theta(1-\theta) k}} .
\end{aligned}
$$

It is not difficult to check that

$$
\begin{gathered}
\lambda_{r, k, n}=(n-k+1) f(r ; k, p)=l_{n}\left(1+O\left(\frac{\rho^{2}+1}{k}\right)\right), \\
\left|\mathrm{e}^{-\lambda_{r, k, n}}-\mathrm{e}^{-l_{n}}\right|=\mathrm{e}^{-l_{n}}\left|1-\mathrm{e}^{l_{n}-\lambda_{r, k, n}}\right|=O\left(l_{n}-\lambda_{r, k, n}\right)=O\left(\frac{\rho^{2}+1}{k}\right) .
\end{gathered}
$$

On the other hand, the upper bound provided for $\left|F_{n, k}(r)-\mathrm{e}^{-\lambda_{r, k, n}}\right|$ by Corollary 1 takes the asymptotic form

$$
\begin{aligned}
l_{n}(2 k-1) & \frac{q \theta \mathrm{e}^{-k H(\theta, p)-\rho h(\theta, p)}}{\sqrt{2 \pi \theta(1-\theta) k}}+3 l_{n} k \frac{(\theta-p) \mathrm{e}^{-k H(\theta, p)-\rho h(\theta, p)}}{\sqrt{2 \pi \theta(1-\theta) k}} \\
& +\left(l_{n}+2\right) \frac{(\theta-p) k \mathrm{e}^{-k H(\theta, p)-\rho h(\theta, p)}}{\sqrt{2 \pi \theta(1-\theta) k}} \\
\sim & \frac{k \mathrm{e}^{-k H(\theta, p)-\rho h(\theta, p)}}{\sqrt{2 \pi \theta(1-\theta) k}}\left(l_{n}(6 \theta-2 \theta p-4 p)+2(\theta-p)\right) \\
= & O\left(\sqrt{k} \mathrm{e}^{-k H(\theta, p)-\rho h(\theta, p)}\right),
\end{aligned}
$$

and the proof is easily completed by incorporating the above results into (11).

\section{An extreme value theorem for the Erdôs-Rényi statistic}

A substantial literature on asymptotic results has been published under the heading of ErdősRényi laws. A nice collection of results of this type may be found in [5] and the references cited therein.

Let $Y_{1}, Y_{2}, \ldots$ be a sequence of i.i.d. RVs with $\mathrm{E}\left(Y_{i}\right)=0, i=1,2, \ldots$, and define the statistic

$$
U_{n}=\max _{1 \leq i \leq n-k+1} \sum_{j=i}^{i+k-1} Y_{j},
$$

which measures the maximum of the moving sums $\sum_{j=i}^{i+k-1} Y_{j}, i=1,2, \ldots, n-k+1$. The classical Erdős-Rényi theorem [6] states that if $k \equiv k_{n}=\lfloor c \ln n\rfloor$ for $c>0$, then $U_{n} / a k_{n} \rightarrow 1$ almost surely for a large class of distributions of $Y_{i}$ (here $a>0$ is a number 
depending on the distribution of $Y_{i}$ and the constant $c$ ). Deheuvels and Devroye [5] derived an extreme value result for the same statistic. More specifically, they proved that if the $Y_{i}$ obey any nonlattice distribution and $k \equiv k_{n}=\lfloor c \ln n\rfloor, c>0$, then

$$
\lim _{n \rightarrow \infty} \mathrm{P}\left(\frac{U_{n}-b_{n}}{a_{n}} \leq x\right)=\Lambda(x), \quad x \in \mathbb{R},
$$

where $\Lambda(x)=\exp \left(-\mathrm{e}^{-x}\right)$ is the cumulative distribution function of the Gumbel distribution and $a_{n}>0$ and $b_{n} \in \mathbb{R}$ are appropriate normalizing constants.

We shall now exploit Theorem 3 to establish a similar extreme value result when the sequence of i.i.d. RVs are binary Bernoulli variables (lattice distribution with span 1).

Theorem 4. Let $X_{1}, X_{2}, \ldots$ be a sequence of i.i.d. binary $R V$ s with constant success probabilities $p=\mathrm{P}\left(X_{1}=1\right)=1-\mathrm{P}\left(X_{1}=0\right)$, let $\theta \in(p, 1)$, and let $k \equiv k_{n}=\lfloor\ln n / H(\theta, p)\rfloor$. If $\Lambda(x)=\exp \left(-\mathrm{e}^{-x}\right)$ denotes the cumulative distribution function of the Gumbel distribution and

$$
b_{n}=k_{n} \theta+\frac{1}{h(\theta, p)} \ln \frac{n(\theta-p) \mathrm{e}^{-k_{n} H(\theta, p)}}{\sqrt{2 \pi \theta(1-\theta) k_{n}}},
$$

then, for the discrete scan statistic $S_{n, k}=\max _{1 \leq i \leq n-k+1} \sum_{j=i}^{i+k-1} X_{j}$, we have

$$
\lim _{n \rightarrow \infty}\left[\mathrm{P}\left(\frac{S_{n, k}-b_{n}}{1 / h(\theta, p)}<y\right)-\Lambda\left(y-\varepsilon_{n}(y) h(\theta, p)\right)\right]=0,
$$

where

$$
\varepsilon_{n}(y)=\left(b_{n}+\frac{y}{h(\theta, p)}\right)-\left\lfloor b_{n}+\frac{y}{h(\theta, p)}\right\rfloor .
$$

Moreover, the rate of convergence in (12) is of order $O\left((\ln k)^{2} / k\right)$.

Proof. On introducing the notation

$$
r_{n}(y)=b_{n}+\frac{y}{h(\theta, p)},
$$

we may express the probability appearing in (12) as

$$
\mathrm{P}\left(S_{n, k}<\left\lfloor r_{n}(y)\right\rfloor\right)=\mathrm{P}\left(S_{n, k}<r_{n}\right), \quad r_{n}=\left\lfloor r_{n}(y)\right\rfloor .
$$

In order to make use of Theorem 3, observe that $\varepsilon_{n}(y)=r_{n}(y)-\left\lfloor r_{n}(y)\right\rfloor$ while

$$
\begin{aligned}
r_{n}-\theta k_{n} & =r_{n}(y)-\varepsilon_{n}(y)-k_{n} \theta \\
& =\frac{1}{h(\theta, p)}\left[y+\ln \frac{\theta-p}{\sqrt{2 \pi \theta(1-\theta)}}-\frac{1}{2} \ln k_{n}+\left(\frac{\ln n}{H(\theta, p)}-k_{n}\right) H(\theta, p)\right]-\varepsilon_{n}(y) \\
& =O\left(\ln k_{n}\right) \\
& =o(\sqrt{n}) .
\end{aligned}
$$

Moreover, note that both $r_{n}$ and $k_{n}$ tend to $\infty$ as $n \rightarrow \infty$, while the quantity $l_{n}$ used in Theorem 3 takes the form

$$
\begin{aligned}
l_{n} & =n \frac{(\theta-p) \mathrm{e}^{-k_{n} H(\theta, p)}}{\sqrt{2 \pi \theta(1-\theta) k_{n}}} \exp \left(-y-\ln \frac{n(\theta-p) \mathrm{e}^{-k_{n} H(\theta, p)}}{\sqrt{2 \pi \theta(1-\theta) k_{n}}}+\varepsilon_{n}(y) h(\theta, p)\right) \\
& =\mathrm{e}^{-y+\varepsilon_{n}(y) h(\theta, p)} .
\end{aligned}
$$


Since $l_{n}$ is bounded (note that $\varepsilon_{n}(y) \in[0,1)$ ), a direct application of Theorem 3 yields the limiting expression (12). The rate of convergence is given by

$$
O\left(\frac{\left(r_{n}-\theta k_{n}\right)^{2}+1}{k}\right)=O\left(\frac{(\ln k)^{2}}{k}\right) .
$$

It is worth mentioning that the above asymptotic result can be written in the equivalent form

$$
\begin{gathered}
\mathrm{P}\left(\left(U_{n}-k_{n}(\theta-p)\right) h(\theta, p)+\frac{1}{2} \ln k_{n}-\ln \frac{n p(1-\theta)(\theta-p) \mathrm{e}^{-k_{n} H(\theta, p)}}{(1-p) \theta \sqrt{2 \pi \theta(1-\theta)}} \leq y\right) \\
=\exp \left(-\exp \left(-y+\varepsilon_{n}(y) \ln \frac{(1-p) \theta}{p(1-\theta)}\right)\right)+O\left(\frac{(\ln k)^{2}}{k}\right),
\end{gathered}
$$

where $U_{n}=\max _{1 \leq i \leq n-k+1} \sum_{j=i}^{i+k-1}\left(X_{j}-p\right)$. The last expression is almost the same as that of Theorem 6 of [5] (when applied to Bernoulli variables), the only difference being in the additional oscillating term $\varepsilon_{n}(y) \ln ((1-p) \theta / p(1-\theta))$ appearing on the left-hand side of (13). This is because the result of [5] holds only for nonlattice distributions, whereas (our) Theorem 4 refers to the Bernoulli distribution. Apparently, $U_{n}$ does not belong to the domain of attraction of an extreme value distribution in the case of Bernoulli RVs, and the same will hold for all lattice distributions. Nevertheless, if we can determine appropriate sequences $\left\{n_{i} \in \mathbb{N}\right\}$, such that $\varepsilon_{n_{i}}(y) \rightarrow \varepsilon(y)$ as $i \rightarrow \infty$ for every $y$, we may obtain an extreme value distribution for the (normalized) $U_{n_{i}}, i=1,2, \ldots$, of the form

$$
\exp \left(-\exp \left(-y+\varepsilon(y) \ln \frac{(1-p) \theta}{p(1-\theta)}\right)\right)
$$

\section{Numerical results}

In the previous sections, three different approximations were developed for the cumulative distribution function, $F_{n, k}(r)=\mathrm{P}\left(S_{n, k}<r\right)$, of the discrete scan statistic $S_{n, k}$. It is worth mentioning that the expected number of successes within a scanning window of length $k$ is $k p$ and, therefore, that $F_{n, k}(r)=\mathrm{P}\left(S_{n, k}<r\right) \approx 0$ when $r \leq p k$. For this reason, in the sequel we shall assume that $r>p k$. According to Corollary $1, F_{n, k}(r)$ can be approximated by the quantity

$$
F_{1}(n, k, r ; p)=\exp (-\lambda)=\exp (-(n-k+1) f(r ; k, p)), \quad r>k p,
$$

with $f(r ; k, p)$ as given in (4).

Theorem 3 states that the asymptotic behavior of $F_{n, k}(r)$ can be investigated with the use of the expression $\exp \left(-l_{n}\right)$. With $\theta=r / k$, the quantity $\exp \left(-l_{n}\right)$ reduces to

$$
F_{2}(n, k, r ; p)=\exp \left(-n \frac{(r / k-p) \mathrm{e}^{-k H(r / k, p)}}{\sqrt{2 \pi(r / k)(1-r / k) k}}\right), \quad r>k p,
$$

with $H(\theta, p)$ as given in (7).

Finally, Theorem 4 offers a third asymptotic approximation for $F_{n, k}(r)$ in terms of the cumulative distribution function of the Gumbel distribution. This third approximation converges quite slowly (especially when $r$ is not very close to $\theta k$ ), a fact that holds for the majority of Erdős-Rényi-type laws as well. Therefore, this result is primarily of theoretical interest. 
The other two expressions, $F_{1}(n, k, r ; p)$ and $F_{2}(n, k, r ; p)$, can be used to obtain quite reasonable approximations for the cumulative distribution $F_{n, k}(r)$.

Were we interested in the expected value of $S_{n, k}$, we could make use of the well-known formula

$$
\mathrm{E}\left(S_{n, k}\right)=\sum_{r=1}^{\infty} \mathrm{P}\left(S_{n, k} \geq r\right)=\sum_{r=1}^{\infty}\left(1-\mathrm{P}\left(S_{n, k}<r\right)\right)=\sum_{r=1}^{\infty}\left(1-F_{n, k}(r)\right),
$$

which, on taking into account the facts that $F_{n, k}(r) \approx 0$ for $r \leq k p$ and $F_{n, k}(r)=1$ for $r>k$, yields

$$
\mathrm{E}\left(S_{n, k}\right) \approx r_{0}+\sum_{r=r_{0}+1}^{k}\left(1-F_{n, k}(r)\right), \quad r_{0}=\lfloor k p\rfloor .
$$

Next, by replacing $F_{n, k}(r)$ by $F_{1}(n, k, r ; p)$ and then $F_{2}(n, k, r ; p)$, we may write

$$
\mathrm{E}\left(S_{n, k}\right) \approx r_{0}+\sum_{r=r_{0}+1}^{r_{1}}\left(1-\mathrm{e}^{-(n-k+1) f(r ; k, p)}\right)
$$

and, respectively,

$$
\mathrm{E}\left(S_{n, k}\right) \approx r_{0}+\sum_{r=r_{0}+1}^{r_{1}}\left(1-\exp \left(-n \frac{(r / k-p) \mathrm{e}^{-k H(r / k, p)}}{\sqrt{2 \pi(r / k)(1-r / k) k}}\right)\right),
$$

with the summations terminating whenever the approximate value for $F_{n, k}(r)$ is almost 1 (i.e. $F_{r, k}\left(r_{1}\right) \approx 1$ ). In the same fashion, we could also use the expression

$$
\mathrm{E}\left(S_{n, k}^{m}\right)=\sum_{r=1}^{k}\left(r^{m}-(r-1)^{m}\right) \mathrm{P}\left(S_{n, k} \geq r\right), \quad m=1,2, \ldots,
$$

to obtain reasonable and computationally tractable approximations for the higher moments of $S_{n, k}$.

In Tables 1-6 we provide Monte Carlo estimations (denoted Sim) of the exact values of $F_{n, k}(r)=\mathrm{P}\left(S_{n, k}<r\right)$ and $\mathrm{E}\left(S_{n, k}\right)$ along with the respective approximations, for a variety

TABLE 1: $n=1000, k=30, p=0.5$.

\begin{tabular}{cccccc}
\hline$r$ & Sim & $F_{1}$ & $U B$ & $F_{2}$ & $Q_{L}^{\prime}$ \\
\hline 20 & 0.0030 & 0.010481 & 5.814920 & 0.009012 & 0.003081 \\
21 & 0.0543 & 0.074823 & 1.838730 & 0.067685 & 0.053611 \\
22 & 0.2703 & 0.290652 & 0.445214 & 0.276236 & 0.270714 \\
23 & 0.6039 & 0.612020 & 0.089468 & 0.599248 & 0.604788 \\
24 & 0.8507 & 0.851214 & 0.017160 & 0.845072 & 0.849814 \\
25 & 0.9580 & 0.957953 & 0.003415 & 0.955991 & 0.957749 \\
26 & 0.9904 & 0.990954 & 0.000646 & 0.990486 & 0.990927 \\
27 & 0.9985 & 0.998532 & 0.000100 & 0.998446 & 0.998529 \\
& $\mathrm{E}\left(S_{n, k}\right)=22.272$ & $\mathrm{E}\left(S_{n, k}\right)=22.212$ & - & $\mathrm{E}\left(S_{n, k}\right)=22.256$ & - \\
\hline
\end{tabular}


TABLE 2: $n=10000, k=100, p=0.5$.

\begin{tabular}{cccccc}
\hline$r$ & $\operatorname{Sim}$ & $F_{1}$ & $U B$ & $F_{2}$ & $Q_{L}^{\prime}$ \\
\hline 61 & 0.0002 & 0.000426 & 6.211840 & 0.000393 & 0.000166 \\
63 & 0.0259 & 0.030981 & 1.196450 & 0.029690 & 0.026104 \\
65 & 0.2697 & 0.277177 & 0.172825 & 0.272680 & 0.270811 \\
67 & 0.6742 & 0.676179 & 0.021687 & 0.672762 & 0.674519 \\
69 & 0.9056 & 0.906263 & 0.003008 & 0.905096 & 0.906049 \\
71 & 0.9795 & 0.979849 & 0.000487 & 0.979585 & 0.979825 \\
73 & 0.9966 & 0.996562 & 0.000077 & 0.996515 & 0.996559 \\
75 & 0.9995 & 0.999527 & 0.000010 & 0.999520 & 0.999526 \\
& $\mathrm{E}\left(S_{n, k}\right)=65.80$ & $\mathrm{E}\left(S_{n, k}\right)=65.766$ & - & $\mathrm{E}\left(S_{n, k}\right)=65.788$ & - \\
\hline
\end{tabular}

TABLE 3: $n=100000, k=1000, p=0.5$.

\begin{tabular}{cccccc}
\hline$r$ & Sim & $F_{1}$ & $U B$ & $F_{2}$ & $Q_{L}^{\prime}$ \\
\hline 539 & 0.0062 & 0.009528 & 4.078320 & 0.009118 & 0.006942 \\
543 & 0.0577 & 0.070025 & 1.270740 & 0.068191 & 0.063361 \\
547 & 0.2288 & 0.243519 & 0.353627 & 0.240034 & 0.236715 \\
551 & 0.4878 & 0.497304 & 0.090433 & 0.493741 & 0.493687 \\
555 & 0.7169 & 0.724794 & 0.022406 & 0.722389 & 0.723520 \\
559 & 0.8669 & 0.870856 & 0.005799 & 0.869612 & 0.870493 \\
563 & 0.9444 & 0.946087 & 0.001658 & 0.945544 & 0.945989 \\
567 & 0.9796 & 0.979482 & 0.000518 & 0.979272 & 0.979455 \\
571 & 0.9928 & 0.992786 & 0.000166 & 0.992711 & 0.992778 \\
& $\mathrm{E}\left(S_{n, k}\right)=551.55$ & $\mathrm{E}\left(S_{n, k}\right)=551.17$ & - & $\mathrm{E}\left(S_{n, k}\right)=551.23$ & - \\
\hline
\end{tabular}

TABLE 4: $n=100000, k=100, p=0.5$.

\begin{tabular}{cccccc}
\hline$r$ & $\operatorname{Sim}$ & $F_{1}$ & $U B$ & $F_{2}$ & $Q_{L}^{\prime}$ \\
\hline 66 & 0.0004 & 0.000660 & 0.4620750 & 0.000642 & 0.000617 \\
67 & 0.0188 & 0.019289 & 0.1353200 & 0.018994 & 0.018919 \\
68 & 0.1303 & 0.131504 & 0.0372180 & 0.130446 & 0.130825 \\
69 & 0.3728 & 0.370420 & 0.0098961 & 0.368931 & 0.369945 \\
70 & 0.6282 & 0.629420 & 0.0026684 & 0.628218 & 0.629231 \\
71 & 0.8157 & 0.814325 & 0.0007715 & 0.813621 & 0.814269 \\
72 & 0.9199 & 0.916981 & 0.0002460 & 0.916640 & 0.916966 \\
73 & 0.9673 & 0.965843 & 0.0000847 & 0.965696 & 0.965839 \\
74 & 0.9867 & 0.986855 & 0.0000300 & 0.986797 & 0.986854 \\
75 & 0.9947 & 0.995233 & 0.0000105 & 0.995211 & 0.995233 \\
76 & 0.9979 & 0.998367 & 0.0000035 & 0.998359 & 0.998367 \\
& $\mathrm{E}\left(S_{n, k}\right)=69.21$ & $\mathrm{E}\left(S_{n, k}\right)=69.172$ & - & $\mathrm{E}\left(S_{n, k}\right)=69.177$ & - \\
\hline
\end{tabular}


TABLE 5: $n=100000, k=1000, p=0.7$.

\begin{tabular}{cccccc}
\hline$r$ & $\operatorname{Sim}$ & $F_{1}$ & $U B$ & $F_{2}$ & $Q_{L}^{\prime}$ \\
\hline 735 & 0.0028 & 0.006738 & 4.391180 & 0.006420 & 0.004679 \\
739 & 0.0566 & 0.067919 & 1.206990 & 0.066100 & 0.061369 \\
743 & 0.2612 & 0.267633 & 0.287480 & 0.264010 & 0.261151 \\
747 & 0.5516 & 0.554803 & 0.061936 & 0.551410 & 0.551898 \\
751 & 0.7796 & 0.786442 & 0.013116 & 0.784471 & 0.785624 \\
755 & 0.9093 & 0.914489 & 0.003028 & 0.913635 & 0.914301 \\
759 & 0.9661 & 0.970108 & 0.000789 & 0.969800 & 0.970065 \\
763 & 0.9877 & 0.990646 & 0.000218 & 0.990548 & 0.990636 \\
767 & 0.9951 & 0.997350 & 0.000059 & 0.997322 & 0.997347 \\
& $\mathrm{E}\left(S_{n, k}\right)=746.46$ & $\mathrm{E}\left(S_{n, k}\right)=746.28$ & - & $\mathrm{E}\left(S_{n, k}\right)=746.33$ & - \\
\hline \multicolumn{7}{r}{} & & & &
\end{tabular}

TABLe 6: $n=10000, k=100, p=0.7$.

\begin{tabular}{cccccc}
\hline$r$ & $\operatorname{Sim}$ & $F_{1}$ & $U B$ & $F_{2}$ & $Q_{L}^{\prime}$ \\
\hline 81 & 0.0062 & 0.008590 & 2.104690 & 0.008042 & 0.006245 \\
82 & 0.0546 & 0.060537 & 0.730446 & 0.058116 & 0.054386 \\
83 & 0.2081 & 0.215001 & 0.228520 & 0.210098 & 0.208207 \\
84 & 0.4521 & 0.457658 & 0.066394 & 0.452187 & 0.453727 \\
85 & 0.6895 & 0.692245 & 0.018849 & 0.688244 & 0.690780 \\
86 & 0.8502 & 0.852425 & 0.005543 & 0.850228 & 0.852004 \\
87 & 0.9371 & 0.938287 & 0.001731 & 0.937294 & 0.938179 \\
88 & 0.9774 & 0.977020 & 0.000557 & 0.976630 & 0.976992 \\
89 & 0.9920 & 0.992309 & 0.000174 & 0.992173 & 0.992302 \\
90 & 0.9976 & 0.997685 & 0.000051 & 0.997642 & 0.997683 \\
& $\mathrm{E}\left(S_{n, k}\right)=86.56$ & $\mathrm{E}\left(S_{n, k}\right)=86.565$ & - & $\mathrm{E}\left(S_{n, k}\right)=86.573$ & - \\
\hline
\end{tabular}

of the parameters $n, k$, and $r$, and for $p=0.5,0.7$. The quantity $U B$ is a bound for the discrepancy between $F_{n, k}(r)$ and $F_{1}$ (see Corollary 1). It is clear that, as $n, k$, and $r$ increase, the quality of the approximation of $F_{n, k}(r)$ improves substantially. For comparison reasons we have also included in the table a third approximation (denoted $Q_{L}^{\prime}$ ) for the same quantity which was suggested by Glaz et al. [12, p. 45, Equation (4.3)]. Note that $Q_{L}^{\prime}$ also provides very accurate approximations, especially for large values of $n, k$, and $r$. However, the computational difficulty of evaluating $Q_{L}^{\prime}$ is much higher (as compared to $F_{1}(n, k, r ; p)$ and especially $\left.F_{2}(n, k, r ; p)\right)$; in addition, no estimate is available for the convergence rate of the approximation established with the use of $Q_{L}^{\prime}$.

It should be stressed that the arguments used to derive $Q_{L}^{\prime}$ do not offer any clue as to how the approximation error can be bounded. Corollary 1, on the contrary, offers an explicit, computationally tractable bound for the discrepancy between $F_{n, k}(r)$ and $F_{1}(n, k, r ; p)$, namely

$$
U B=(2 k-1) \lambda p q b(r-1 ; k-1, p)+3 \lambda k f(r ; k, p)+(\lambda+2)(1-G(r ; k, p)) .
$$

As $r$ increases, the quantity $U B$ becomes extremely small (less than $10^{-4}$ ) and, as a consequence, a very tight interval estimate for $F_{n, k}(r)$ may be developed. Note that the rate of convergence 
for the approximation provided by $F_{2}(n, k, r ; p)$ is also available; however, this cannot be used efficiently to establish interval estimates this good.

In closing, we mention that we could formally write down an exact formula for the distribution of $S_{n, k}$ by embedding the RV of interest in an appropriate Markov chain (see [7] and [1, p. 297]). However, the dimension of the transition probability matrix of the chain becomes extremely large even for moderate values of $r$ and $k$ (it is nearly one billion for the smallest tabulated values, $k=30$ and $r=20$ ), a fact that makes the evaluation unfeasible. In cases where the parameter values lead to intractable computations, the approach taken in this article is of special interest.

\section{References}

[1] Balakrishnan, N. and Koutras, M. V. (2002). Runs and Scans with Applications. John Wiley, Chichester.

[2] Barbour, A. D. and Chryssaphinou, O. (2001). Compound Poisson approximation: a user's guide. Ann. Appl. Prob. 11, 964-1002.

[3] Boutsikas, M. V. And Koutras, M. V. (2001). Compound Poisson approximation for sums of dependent random variables. In Probability and Statistical Models with Applications, eds C. A. Charalambides, M. V. Koutras and N. Balakrishnan, Chapman and Hall/CRC Press, Boca Raton, FL, pp. 63-86.

[4] Boutsikas, M. V. And Koutras, M. V. (2002). Modeling claim exceedances over thresholds. Insurance Math. Econom. 30, 67-83.

[5] Deheuvels, P. and Devroye, L. (1987). Limit laws of Erdős-Rényi-Shepp type. Ann. Prob. 15, $1363-1386$.

[6] ERdôs, P. And RÉnYI, A. (1970). On a new law of large numbers. J. Anal. Math. 23, 103-111.

[7] Fu, J. C. (2001). Distribution of the scan statistic for a sequence of bistate trials. J. Appl. Prob. 38, $908-916$.

[8] Fu, J. C. AND Lou, W. Y. W. (2003). Distribution Theory of Runs and Patterns and Its Applications. A Finite Markov Chain Imbedding Approach. World Scientific, Singapore.

[9] Glaz, J. and Balakrishnan, N. (eds) (1999). Scan Statistics and Applications. Birkhäuser, Boston, MA.

[10] Glaz, J. and Naus, J. I. (1991). Tight bounds and approximations for scan statistic probabilities for discrete data. Ann. Appl. Prob. 1, 306-318.

[11] Glaz, J. And Zhang, Z. (2004). Multiple window discrete scan statistics. J. Appl. Stat. 31, 967-980.

[12] Glaz, J., Naus, J. and Wallenstein, S. (2001). Scan Statistics. Springer, New York.

[13] HöGLund, T. (1979). A unified formulation of the central limit theorem for small and large deviations from the mean. Z. Wahrscheinlichkeitsth. 49, 105-117.

[14] Petrov, V. V. (1965). On the probabilities of large deviations for sums of random variables. Theory Prob. Appl. 10, 287-298.

[15] Roos, M. (1994). Stein's method for compound Poisson approximation: the local approach. Ann. Appl. Prob. 4, 1177-1187. 